\title{
External arguments in the multiple-spiral medallions of the Mandelbrot set
}

\author{
M. Romera ${ }^{*}$, G. Pastor, G. Alvarez, and F. Montoya \\ Instituto de Física Aplicada, Consejo Superior de Investigaciones Científicas \\ Serrano 144, 28006 Madrid, Spain
}

\begin{abstract}
The discs of a small copy of the Mandelbrot set $\mathcal{M}$ have antennas or shrubs as the discs of the whole $\mathcal{M}$, with the obvious difference that the shrubs of the small copy are prolonged with what we name tendrils. There are beautiful decorations in the tendrils, named by us multiple-spiral medallions that are formed by an infinite number of baby Mandelbrot sets. The computer graphics and the external arguments theory are two invaluable tools to study the structure of these decorations. In this work we conjecture that the baby Mandelbrot sets of a multiple-spiral medallion have a "parent" and a "gene". This conjecture allows us to write the binary expansions of the external arguments of the baby Mandelbrot sets in an easy and structured way.

PACS number: 05.45.Df
\end{abstract}

Keywords: Mandelbrot set, cauliflowers, external arguments, binary expansions, shrubs.

\section{Introduction}

The multiple-spiral medallions are beautiful structures of the Mandelbrot set that are very difficult to discover using only mathematical tools. However, they are evident using computer graphics. In this work we use computer graphics to analyze the structure of the multiple-spiral medallions, and the external arguments theory to explain how they are mathematically organized.

When the boundary of the Mandelbrot set $\mathcal{M}$ is explored, crossing the filaments or shrubs of one of its discs, small copies of $\mathcal{M}$ that we call midgets are found out. By increasing the magnification of the observation tool, the boundary of one of the midgets

\footnotetext{
* Corresponding author. Tel.: +34-915631284; fax: +34-914117651.

E-mail address: miguel@iec.csic.es (M. Romera)
} 
can be explored. Now, midget shrubs are extended in tendrils. These tendrils are born of both the tips of the midget shrubs and the cusp of the midget cardioid. In addition, small objects embedded in the tendrils, which can not be seen with the current magnification, are glimpsed. By increasing the magnification, these objects, that we call multiple-spiral medallions and that are a generalization of the known cauliflowers located at the tendrils of the cusp of the midget cardioid [1], can be explored. A multiple-spiral medallion is formed by an infinite number of baby Mandelbrot sets arranged in spirals of multiple branches. By using the Douady and Hubbard external arguments theory, in this paper we state two conjectures that allow to formulate the external arguments of the baby Mandelbrot sets of a multiple-spiral medallion as a function of the external arguments of the root points of both a midget (that we call parent) and a disc (that we call gene). According to Douady, Buff, Devaney and Sentenac [1] "Baby Mandelbrot sets are born in cauliflowers". Colloquially, we can complete the statement of Douady et al. with "... and baby Mandelbrot sets have a parent and a gene that are the same for each cauliflower (multiple-spiral medallion)".

In the 80 's Douady and Hubbard were the authors of the external argument theory in the Mandelbrot set $\mathcal{M}[2,3]$. Douady divulged this theory [4] by considering a capacitor made of a hollow metallic cylinder whose axis is an aluminum bar shaped in such a way that its cross-section is $\mathcal{M}$. Under proper conditions, an electric field is obtained that defines equipotential lines enclosing $\mathcal{M}$. Following the electric field, one gets field-lines, called the external rays of $\mathcal{M}$. Each external ray starts at a point $x$ on the boundary of $\mathcal{M}$, and reaches a point $y$ of the cross section of the cylinder. The position of $y$ is identified by an angle, called the external argument of $x$ with respect to $\mathcal{M}$

In Fig. 1 the Mandelbrot set is shown with some of its equipotential lines and external rays. The rational numbers in the figure correspond to the values of the external arguments of the points of $\mathcal{M}$ where the external rays land. As is well known the unity for external arguments is the whole turn, not the radian. It is often useful to consider the binary expansion (sequence of binary digits) of a rational external argument [5]. So, we 
have $\frac{1}{15}=. \overline{0001}$ and $\frac{1}{6}=.0 \overline{01}$. As is known, the binary expansion of a rational number with odd denominator is periodic, and the binary expansion of a rational number with even denominator is preperiodic.

A key point in Douady and Hubbard's study of the Mandelbrot set is the theorem that every parabolic point, $c \neq 1 / 4$, in $\mathcal{M}$ is the landing point for exactly two external rays with external arguments which are periodic under doubling [6]. As is well known, a point is parabolic if and only if it has a periodic orbit with some root of unity as multiplier; i.e., it is a cusp point or a tangent point in $\mathcal{M}$. The external arguments corresponding to the two external rays landing at the same parabolic point must have the same kneading sequence [5]. As is known [5], the kneading sequence of an external argument $K(\theta)$ is given by

$$
n \text {-th entry of } K(\theta):= \begin{cases}\mathrm{L} & \text { if } \left.2^{n} \theta \in\right] \frac{\theta}{2}, \frac{\theta+1}{2}[\bmod 1 \\ \mathrm{R} & \text { if } \left.2^{n} \theta \in\right] \frac{\theta+1}{2}, \frac{\theta}{2}[\bmod 1 \\ * & \text { if } 2^{n} \theta=\frac{\theta}{2} \bmod 1 \\ * & \text { if } 2^{n} \theta=\frac{\theta+1}{2} \bmod 1 .\end{cases}
$$

For example, the external arguments of the two external rays landing at the tangent point of the main cardioid with the upper period-4 disc of $\mathcal{M}$ are $\frac{1}{15}=. \overline{0001}$ and $\frac{2}{15}=. \overline{0010}$ (see Fig. 1) and we have $K\left(\frac{1}{15}\right)=K\left(\frac{2}{15}\right)=$ LLL $*$. In this work we will write the binary expansions of the external arguments of the two external rays landing at a parabolic point as the pair $\left(\overline{\theta_{1}}, \overline{\theta_{2}}\right)$ with $. \overline{\theta_{1}}<. \overline{\theta_{2}}$.

\section{Multiple-spiral medallions}

As is well known, the discs attached to the main cardioid of $\mathcal{M}$ have antennas [7], filaments [8] or shrubs [9]. In Fig. 2 (a) we can see a general view of $\mathcal{M}$ and the shrub (1/3) i.e. the part of the Mandelbrot set attached to the Myrberg-Feigenbaum point of the disc with rotation number $1 / 3$ is pointed out [7]. 
We name midget to a small copy of $\mathcal{M}$ in $\mathcal{M}$. Let us consider the period-4 midget representative of the branch 1 of the shrub (1/3) [9] that is inside the small square of Fig. 2 (a). For our convenience, in Fig. 2 (b) we show a magnification of this midget up to a similar size to $\mathcal{M}$ of Fig. 2 (a). The two external rays landing at the cusp of the cardioid of the midget have external arguments with binary expansions $(\overline{0011}, \overline{0100})$. As we can see in Fig. 2 (b) the discs attached to the cardioid of the midget have similar shrubs to the Fig. 2 (a), but with the particularity that they are prolonged in what we name tendrils, in such a manner that a tendril is just born at each one of the tips of the midget shrubs. Inside a shrub, the tendril that is born in the first tip [9] is the longest, and the tendril that is born in the last tip [9] is the shortest. On the other hand, there are two very notorious tendrils that emerge from both the tip of the midget main antenna and the cusp of the midget cardioid. Note that the second tendril is the only one that is born at a parabolic point of the midget. Obviously, these two tendrils are a part of the branch of the shrub that supports the midget itself that is the branch 1 of the shrub (1/3) [9].

The midgets located at the tendrils are surrounded by beautiful decorations with central symmetry. In Fig. 3 we show a detail of the tendril from the cusp of the midget $(\overline{0011}, \overline{.0100})$. As is well known, this midget is the biggest of all the midgets located out of the real axis of $\mathcal{M}$ and, for convenience, we have used this midget to make most of the numerical simulations in this work.

According to Douady et al. [1], when one looks closely to $\mathcal{M}$ at the neighborhood of the cusp point of a midget cardioid $c_{0} \neq 1 / 4$, one can observe a sequence of small copies of $\mathcal{M}$ in $\mathcal{M}$, baby Mandelbrot sets, tending to the cusp point. In Fig. 3 no sequence of baby Mandelbrot sets is detected, due to its small size, but however we can observe a sequence of decorations $a, b, c, d, \ldots$ named imploded cauliflowers [1]. In the limit, when the size of decorations tends to cero, the scaling constant of these decorations is equal to unity (they are equally spaced and they have the same size) as it was found in another context [10]. Baby Mandelbrot sets are also midgets, but in this work we will use "midget" to designate a generic copy of $\mathcal{M}$ and 
we will use "baby Mandelbrot set" to designate a copy of $\mathcal{M}$ that is inside of a decoration located in a tendril of the midget. As is known, cauliflowers present a binary structure with infinite levels [11]. Note, as seen in Fig. 3, that there also are cauliflowers on the tendrils of the shrubs of the discs that converge on the cusp of the midget cardioid as the series $m, n, o, \ldots$ or $p, q, r, \ldots$

In Fig. 4 (a) the tendrils of the shrubs converging at the tangent point of the cardioid of the midget $(\overline{.0011}, . \overline{0100})$ with the disc of rotation number $1 / 2$, near the parabolic point $(\overline{.00110100} . \overline{01000011})$, are shown. As can be seen in the magnification inside a circle, the midgets of these tendrils are also surrounded by decorations according to [12]. Likewise, in Fig. 4 (b) the tendrils of the shrubs converging at the tangent point of the cardioid of the midget $(\overline{.0011}, \overline{.0100})$ with the disc of rotation number $1 / 3$, near the parabolic point $(\overline{.001100110100} . \overline{001101000011})$, are shown. As can be seen in the magnification inside a circle, the midgets of these tendrils also are surrounded by decorations.

In Fig. 5 we can see with more detail each one of the decorations corresponding to Figs. 3 and 4. These decorations belong to the tendrils of the midget $(\overline{.0011}, \overline{.0100})$ but similar decorations appear in the corresponding tendrils of others midgets. We name multiple-spiral medallions to these decorations due to its shape. Near the cusp of the midget there are cauliflowers $[1,11]$ that we name single-spiral medallions. Near the tangent point of the midget cardioid with the disc of rotation number $1 / 2$ there are embedded Julia sets [12] that we name double-spiral medallions. Near the tangent point of the midget cardioid with one of its discs of rotation number $1 / 3$ there are decorations that we name triple-spiral medallions. And so on. Also note that there are decorations near the midget main antenna tip, although in this case we can not see spirals but linearlike structures that we name carrots or non-spiral medallions.

As is pointed out in [11] a Douady cauliflower, i.e. a single-spiral medallion, can hardly be seen as a medallion when the period of the central baby Mandelbrot set is low (see, for example, the single-spiral medallion with central baby Mandelbrot set $\left(\overline{p_{1} g_{1}}, \overline{p_{2} g_{2}}\right)$ at the lower right corner of Fig. 9). The same occurs with the double, 
triple...-spiral medallions. However, although the spirals can be difficult to see, a multiple-spiral medallion with central baby Mandelbrot set of low period has the same structure that one with central baby Mandelbrot set of higher period, as we can see in Fig. 6.

The complexity of the Mandelbrot set is so extraordinary that any attempt to order its hyperbolic components must necessarily be partial, above all when the approach is completely experimental by using computer graphics. In this case there are infinite decorations on a tendril and we will only consider the structural ones which are the biggest. Examples of structural multiple-spiral medallions are the series $a, b, c, d, \ldots$ $m, n, o, \ldots$ and $p, q, r, \ldots$ (Fig. 3).

\section{Binary expansions of the central baby Mandelbrot set of a multiple-spiral medallion}

Let us consider a multiple-spiral medallion on a tendril of a midget that we name parent $\left(\overline{p_{1}}, \overline{p_{2}}\right)$. The parent is located at a branch of the shrub [9] that emerges from the Myrberg-Feigenbaum point of the period doubling cascade of a disc that we name gene $\left(\overline{g_{1}}, \overline{g_{2}}\right)$. Note that $. \overline{g_{1}}<. \overline{p_{1}}<\cdot \overline{p_{2}}<. \overline{g_{2}}$ because the parent is inside the wake of the gene (see Fig. 7). Let $\overline{p_{*}}$ be $\overline{p_{1}}$ or $\cdot \overline{p_{2}}$ and let $\cdot \overline{g_{*}}$ be $\overline{g_{1}}$ or $\cdot \overline{g_{2}}$. According to our observations we enunciate the following

\section{Conjecture 1}

The binary expansions of the central baby Mandelbrot set $\left(\overline{b_{1}}, \overline{b_{2}}\right)$ of a multiple-spiral medallion can be obtained by a composition of the binary expansions of the parent and the gene in the form

$$
\left(\overline{b_{1}}, \overline{b_{2}}\right)=(\underbrace{\overline{p_{*} p_{*} \ldots p_{*}} g_{*}}_{i}, \underbrace{\overline{p_{*} p_{*} \ldots p_{*}} g_{*}}_{i}) \text {, }
$$

with the ordering of Table I. The index $i$ is the number of $p$ 's before $g$. 
Let us note that Table I is strongly ordered in the sense we will see next, and therefore it is immediate to find the binary expansions of the central baby Mandelbrot sets of the multiple-spiral medallions. For example, let us consider the case $i=3$. Firstly, we write the binary-ordered series of $2^{4}$ binary expansions (1) $\overline{p_{1} p_{1} p_{1} g_{1}},(2)$ $\overline{p_{1} p_{1} p_{1} g_{2}}$, (3) $\overline{p_{1} p_{1} p_{2} g_{1}}, \ldots$ (15).$\overline{p_{2} p_{2} p_{2} g_{1}}$, (16) $\overline{p_{2} p_{2} p_{2} g_{2}}$. Secondly, we reorder cyclically these binary expansions in pairs according to Fig. 8; i.e., we form the pairs of binary expansions $(1,16),(2,3),(4,5), \ldots(14,15)$. Obviously, by generalizing, firstly

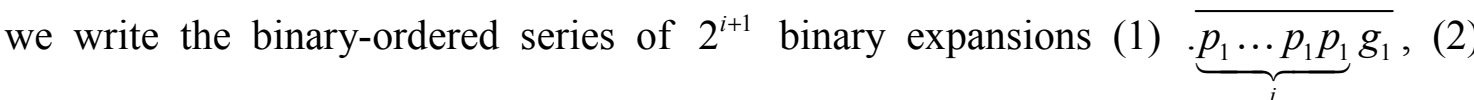
$\underbrace{\overline{p_{1} \ldots p_{1} p_{1}} g_{2}}_{i}$, (3) $\underbrace{\overline{p_{1} \ldots p_{1} p_{2}} g_{1}}_{i}, \ldots\left(2^{i+1}-1\right) \cdot \underbrace{\overline{p_{2} \ldots p_{2} p_{2}} g_{1}}_{i},\left(2^{i+1}\right) \cdot \underbrace{\overline{p_{2} \ldots p_{2} p_{2}} g_{2}}_{i}$; and secondly, we form the pairs of binary expansions $\left(1,2^{i+1}\right),(2,3),(4,5), \ldots$ $\left(2^{i+1}-2,2^{i+1}-1\right)$.

Let us consider two particular cases. Firstly, when the pair of binary expansions of the central baby Mandelbrot set has the form $(\underbrace{\overline{p_{1}^{p_{1} \ldots p_{1}} g_{1}}}_{i}, \underbrace{\overline{p_{2} p_{2} \ldots p_{2}} g_{2}}_{i})=$ $\left(\overline{\bar{p}_{1}^{i} g_{1}}, \overline{\bar{p}_{2}} g_{2}\right)$ the corresponding multiple-spiral medallion is a single-spiral medallion or cauliflower (Fig. 8). Note that the binary expansions $\left(\overline{p_{1} g_{1}}, \overline{p_{2} g_{2}}\right)$, $\left(\overline{p_{1} p_{1} g_{1}}, \overline{p_{2} p_{2} g_{2}}\right)$ and $\left(\overline{p_{1} p_{1} p_{1} g_{1}}, \overline{p_{2} p_{2} p_{2} g_{2}}\right)$ respectively are on the top of the columns of Table I. Secondly, when the pair of binary expansions of the central midget has the form $\left(\overline{p_{1}{\overline{p_{2}}}^{i} g_{2}}, \overline{p_{2}{\overline{p_{1}}}^{i} g_{1}}\right)$ the corresponding multiple-spiral medallion is a nonspiral medallion or carrot (Fig. 8). Note that the binary expansions $\left(. \overline{p_{1} g_{2}}, \overline{p_{2} g_{1}}\right)$, $\left(\overline{p_{1} p_{2} g_{2}}, \overline{p_{2} p_{1} g_{1}}\right)$ and $\left(\overline{p_{1} p_{2} p_{2} g_{2}}, \overline{p_{2} p_{1} p_{1} g_{1}}\right)$ are on the $\left(2^{i-1}+1\right)$ th rows of Table I.

As is known, the external arguments of the tangent point of the midget cardioid $\left(\overline{p_{1}}, \overline{p_{2}}\right)$ with the period-2 disc have binary expansions $\left(\overline{p_{1} p_{2}}, \overline{p_{2} p_{1}}\right)$ [11]. Then, for example, a central baby Mandelbrot set like $\left(\overline{{\overline{p_{1} p_{2}}}^{i} p_{1} g_{2}}, \overline{{\overline{p_{1} p_{2}}}^{i} p_{2} g_{1}}\right)$ is a good 
candidate to be the center of a double-spiral medallion. Likewise, taking into account that the external arguments of the tangent points of the midget cardioid $\left(. \overline{p_{1}}, \overline{p_{2}}\right)$ with the two period-3 discs are $\left(\overline{p_{1} p_{1} p_{2}}, \overline{p_{1} p_{2} p_{1}}\right)$ and $\left(\overline{p_{2} p_{1} p_{2}}, \overline{p_{2} p_{2} p_{1}}\right)$ we can show, for example, that the baby Mandelbrot set $\left(\overline{{\overline{\bar{p}_{1} p_{1} p_{2}}}^{i} p_{1} g_{2}}, \overline{{\overline{p_{1} p_{1} p_{2}}}^{i} p_{2} g_{1}}\right)$ is a good candidate to be the center of a triple-spiral medallion. And so on.

\section{Example 1}

In Fig. 9 the external rays of the central baby Mandelbrot sets of the multiplespiral medallions, $i \leq 3$, corresponding to the parent $\left(. \overline{p_{1}}, \overline{p_{2}}\right)=(\overline{.0011}, \overline{0100})$ and the gene $\left(\overline{g_{1}}, \overline{g_{2}}\right)=(\overline{001}, \overline{010})$ are shown. To see the central baby Mandelbrot sets better, they are surrounded by a small circle. The external rays are drawn starting from a program due to Jung [13].

\section{Example 2}

Let $\left(\overline{p_{1}}, \overline{p_{2}}\right)=(\overline{.01011}, \overline{01100})$ be the parent and let $\left(\overline{g_{1}}, \overline{g_{2}}\right)=$ $(\overline{.010110}, \overline{011001})$ be the gene. We calculate the binary expansions of the central baby Mandelbrot sets in the following cases with $i=10$ :

(a) Non-spiral medallion

$$
\left(\overline{.01011 \overline{01100}^{9} 011001}, \overline{.01100 \overline{01011}^{9} 010110}\right) \text {, }
$$

(b) Single-spiral medallion

$$
\left(\overline{\overline{01011}^{10} 010110}, \overline{\overline{01100}^{10} 011001}\right) \text {, }
$$

(c) Double-spiral medallions

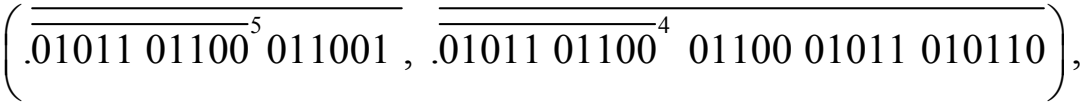

$$
\begin{aligned}
& \left(\overline{\overline{0110001011^{5} 011001}}, \overline{\overline{0110001011} 1^{4} 0110001100010110}\right) \text {, }
\end{aligned}
$$


(d) Triple-spiral medallions

$$
\begin{aligned}
& \left(\overline{\overline{010110101101100}^{3} 01011011001}, \overline{\overline{010110101101100^{3} 01100010110}}\right) \text {, } \\
& \left(\overline{\overline{010110110001011}}^{3} 01011011001, \overline{\overline{010110110001011}^{3} 01100010110}\right) \text {, } \\
& \left(\overline{\overline{.011000101101100}}^{3} 01011011001, \overline{\overline{011000101101100}^{3} 01100010110}\right) \text {, }
\end{aligned}
$$

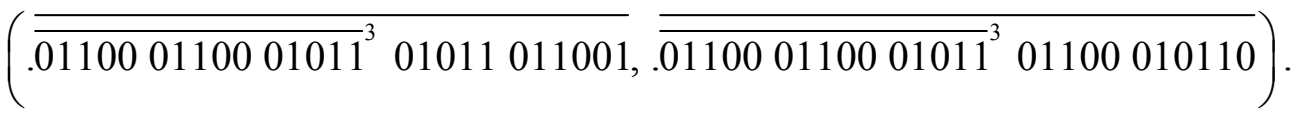

The period of these central baby Mandelbrot sets is 56. Unfortunately, the Jung's program [13] only works up to period 30 and, in this case, we can not draw the external rays to verify the above calculations. However we have verified that in each one of the pairs of binary expansions of this example the two binary expansions have the same kneading sequence.

\section{Binary expansions of the non central baby Mandelbrot sets of a multiple-spiral medallion}

A multiple-spiral medallion is constituted by an infinite number of baby Mandelbrot sets. In this work we will only consider the structural ones corresponding to the biggest baby Mandelbrot sets. The central baby Mandelbrot set is surrounded by the multiple-spiral medallion itself, and the other baby Mandelbrot sets are surrounded by smaller medallions that we name baby medallions (Fig. 5). In the center of a baby medallion there is a baby Mandelbrot set that can be seen with the appropriate magnification.

Let us consider a structural multiple-spiral medallion near a parent $\left(. \overline{p_{1}}, \overline{p_{2}}\right)$ in the wake of a gene $\left(\overline{g_{1}}, \overline{g_{2}}\right)$. Let $\left(\overline{b_{1}}, \overline{b_{2}}\right)$ be the binary expansions of its central baby Mandelbrot set and let $\left(\overline{\beta_{1}}, \overline{\beta_{2}}\right)$ be the binary expansions of one of its structural baby Mandelbrot sets. Let $. \overline{p_{*}}, \overline{g_{*}}, \overline{b_{*}}$, and.$\overline{\beta_{*}}$ be.$\overline{p_{1}}$ or $. \overline{p_{2}}, \overline{g_{1}}$ or $. \overline{g_{2}}, \overline{b_{1}}$ or $\overline{b_{2}}$, and $\overline{\beta_{1}}$ or $\overline{\beta_{2}}$ respectively. According to our observations we enunciate the following 


\section{Conjecture 2}

Each one of the two binary expansions of a non central baby Mandelbrot set of a multiple-spiral medallion can be obtained by a composition of the binary expansions of both the central baby Mandelbrot set and the parent in the form

$$
\overline{\beta_{*}}=\overline{b_{*} \underbrace{p_{*} p_{*} \ldots p_{*}}_{j}} .
$$

The index $j$ is the number of $p$ 's behind $b$.

Taking into account conjecture 1, any of the binary expansions of a non central baby Mandelbrot set can be written as

$$
\overline{\beta_{*}}=\underbrace{\overline{p_{*} p_{*} \ldots p_{*}} g_{*} \underbrace{p_{*} p_{*} \ldots p_{*}}_{j}}_{i} .
$$

For a given value of $j$, we can write $2^{j+1}$ binary expansions.$\overline{\beta_{*}}$ and calculate the corresponding kneading sequences in order to group the $2^{j+1}$ binary expansions in $2^{j}$ pairs having, each one of them, the same kneading sequence. In this way, we can obtain the pairs of binary expansions of all the non central baby Mandelbrot sets of a given multiple-spiral medallion.

Using this procedure we have obtained Tables II and III in the single-spiral and non-spiral medallion cases. Note the clear orderings of the binary expansions of the non central baby Mandelbrot sets in cauliflowers and carrots, due to the fact that these multiple-spiral medallions are on the tendrils that mark the axis of quasi symmetry of the parent. Indeed, if we write the binary-ordered series of $2^{j+1}$ binary expansions (1) $\overline{b_{1} \underbrace{p_{1} \ldots p_{1} p_{1}}_{j}}$, (2) $\overline{b_{1} \underbrace{p_{1} \ldots p_{1} p_{2}}_{j}}$, (3) $\overline{b_{1} \underbrace{p_{1} \ldots p_{2} p_{1}}_{j}}, \ldots\left(2^{j+1}-1\right) \overline{b_{2} \underbrace{p_{2} \ldots p_{2} p_{1}}_{j}},\left(2^{j+1}\right)$ $\overline{b_{2} \underbrace{p_{2} \ldots p_{2} p_{2}}_{j}}$, the column $j$ of Table II is formed by the pairs $(1,2),(3,4),(5,6), \ldots$ $\left(2^{j+1}-1,2^{j+1}\right)$ and the column $j$ of Table III is formed by the pairs $\left(1,2^{j+1}\right),(2$, $\left.2^{j+1}-1\right),\left(3,2^{j+1}-2\right), \ldots\left(2^{j}, 2^{j}+1\right)$. Note that the ordering of the pairs of binary 
expansions in Table II is the same that the ordering of the pairs of binary expansions of Fig. 10 in [11].

If we observe, for example, the top of column 1 of Tables II and III we can see two pairs of binary expansions $\left(\overline{b_{1} p_{1}}, \overline{b_{1} p_{2}}\right)$ and $\left(\overline{b_{1} p_{1}}, \overline{b_{2} p_{2}}\right)$ with a same first value and a different last value, what seems a contradiction. It is due to a notation effect, because the binary expansions of the central baby Mandelbrot sets $\bar{b}_{1}$ are different in cauliflowers and carrots. This apparent contradiction can appear in other cases.

\section{Example 3}

In Fig. 10 some sketches of the location of the binary expansions $\overline{\beta_{*}}=\overline{b_{*} \underbrace{p_{*} p_{*} \ldots p_{*}}_{j}}(j \leq 3)$ of the baby Mandelbrot sets inside of multiple-spiral medallions corresponding to the parent $\left(\overline{p_{1}}, \overline{p_{2}}\right)=(\overline{.0011}, \overline{0100})$ and the gene $\left(\overline{g_{1}}, \overline{g_{2}}\right)=(\overline{.001}, \overline{.010})$ are shown in the cases:

(a) Non-spiral medallion with period-51 central baby Mandelbrot set $\left(\overline{p_{1}{\overline{p_{2}}}^{11} g_{2}}, \overline{p_{2}{\overline{p_{1}}}^{11} g_{1}}\right)$, located at $-0.168988004+1.042370722 i$.

(b) Single-spiral medallion with period-59 central baby Mandelbrot set $\left(\overline{{\overline{p_{1}}}^{14} g_{1}}, \overline{{\overline{p_{2}}}^{14} g_{2}}\right)$, located at $-0.1543869+1.0308295 i$.

(c) Double-spiral medallion with period-135 central baby Mandelbrot set $\left(\overline{{\overline{p_{1} p_{2}}}^{16} p_{1} g_{2}}, \quad \overline{{\overline{p_{1} p_{2}}}^{16} p_{2} g_{1}}\right)$, located at $-0.16092059+1.03663239 i$.

(d) Triple-spiral medallion with period-147 central baby Mandelbrot set $\left(\overline{{\overline{p_{1} p_{1} p_{2}}}^{11} p_{1} p_{1} p_{1} g_{2}}, \quad \overline{{\overline{p_{1} p_{1} p_{2}}}^{11} p_{1} p_{1} p_{2} g_{1}}\right)$, located at $-0.15403780+1.03692215 i$.

\section{Conclusion}

The multiple-spiral medallions (msm) are structures of the Mandelbrot set with central symmetry. They are composed by an infinite number of baby Mandelbrot sets and are a generalization of the known cauliflowers or single-spiral medallions. As we 
show, the msm's are in the tendrils of each midget of the Mandelbrot set. At the same time, as is known, such a midget (parent) is located at a branch of the shrub that emerges from the Myrberg-Feigenbaum point of the period doubling cascade of a disc (gene). In general, a baby Mandelbrot set has a high period. Hence, we think that so far the binary expansions of its external arguments could not be calculated and were difficult to obtain by brute force because of the excessive number of bits. We have conjectured that the pair of binary expansions of the central baby Mandelbrot set of a structural msm can be obtained by an easy composition of the binary expansions pairs of both the parent and the gene. In the same manner, we have conjectured that each one of the binary expansions of a non-central baby Mandelbrot set of a structural msm can be obtained by an easy composition of binary expansions of both the central baby Mandelbrot set and the parent. Subsequently, we can group these binary expansions in pairs having the same kneading sequence. These two conjectures allow us to write the binary expansions of the external arguments of the baby Mandelbrot sets of a multiplespiral medallion in an easy and structured way.

\section{Acknowledgements}

This work was supported by Ministerio de Educación y Ciencia of Spain, research grant SEG2004-02418.

\section{References}

[1] Douady A, Buff X, Devaney RL, Sentenac C. Baby Mandelbrot sets are born in cauliflowers. In: Lei $\mathrm{T}$, editor. The Mandelbrot set, theme and variations. Cambridge: University Press; 2000. p. 19-36.

[2] Douady A, Hubbard JH. Itération des polynômes quadratiques complexes. Comptes Rendus des Séances de L'Académie des Sciences Paris 1982;294:123126.

[3] Douady A. Algorithms for computing angles in the Mandelbrot set. In: Barnsley M, Demko SG, editors. Chaotic dynamics and fractals. New York: Academic Press; 1986. p. 155-168.

[4] Douady A. Julia set and the Mandelbrot set. In: Peitgen H-O, Richter PH, editors. The beauty of fractals. Berlin: Springer; 1986. p. 161-173 (Invited contribution). 
[5] Lau E, Schleicher D. Internal addresses in the Mandelbrot set and irreducibility of polynomials. Stony Brook IMS, preprint 94-19, 1994.

[6] Douady A, Hubbard JH. Étude dynamique des polynômes complexes. Publications mathématiques d'Orsay 84-02 (première partie, 1984) and 85-04 (deuxième partie, 1985).

[7] Devaney RL, Moreno Rocha M. Geometry of the antennas in the Mandelbrot Set. Fractals 2002;10:39-46.

[8] Branner B. The Mandelbrot set. In: Devaney RL, Keen L, editors. Chaos and Fractals. Proceeding of Symposia in Applied Mathematics Vol. 39. New York: American Mathematical Society, 1989. p. 75-105.

[9] Romera M, Pastor G, Alvarez G, Montoya F. Shrubs in the Mandelbrot set ordering. International Journal of Bifurcation and Chaos 2003;13:2279-2300.

[10] Romera M, Pastor G, Montoya F. A scaling constant equal to unity in 1-D quadratic maps. Computers \& Graphics 21:849-857, 1997.

[11] M. Romera, G. Pastor, G. Alvarez and F. Montoya. External arguments of Douady cauliflowers in the Mandelbrot set. Computer \& Graphics 2004;28:437-449.

[12] Munafo RP. Embedded Julia sets. http://www.mrob.com/pub.

[13] Jung W. Mandel.exe. A DOS program available from http:/www.iram.rwthaachen.de/ jung. 
Table I. Binary expansions of the central baby Mandelbrot sets of the multiple-spiral medallions when $i \leq 3$.

$$
\begin{aligned}
& i=1 \quad i=2 \quad i=3 \\
& \left(\overline{. p_{1} g_{1}}, \overline{. p_{2} g_{2}}\right) \quad\left(\overline{p_{1} p_{1} g_{1}}, \overline{p_{2} p_{2} g_{2}}\right) \quad\left(\overline{. p_{1} p_{1} p_{1} g_{1}}, \overline{p_{2} p_{2} p_{2} g_{2}}\right) \\
& \left(\overline{. p_{1} g_{2}}, \overline{p_{2} g_{1}}\right) \quad\left(\overline{p_{1} p_{1} g_{2}}, \overline{p_{1} p_{2} g_{1}}\right) \quad\left(\overline{p_{1} p_{1} p_{1} g_{2}}, \overline{p_{1} p_{1} p_{2} g_{1}}\right) \\
& \left(\overline{p_{1} p_{2} g_{2}}, \overline{p_{2} p_{1} g_{1}}\right) \quad\left(\overline{p_{1} p_{1} p_{2} g_{2}}, \overline{p_{1} p_{2} p_{1} g_{1}}\right) \\
& \left(\overline{p_{2} p_{1} g_{2}}, \overline{p_{2} p_{2} g_{1}}\right) \quad\left(\overline{p_{1} p_{2} p_{1} g_{2}}, \overline{p_{1} p_{2} p_{2} g_{1}}\right) \\
& \left(\overline{p_{1} p_{2} p_{2} g_{2}}, \overline{p_{2} p_{1} p_{1} g_{1}}\right) \\
& \left(\overline{p_{2} p_{1} p_{1} g_{2}}, \overline{p_{2} p_{1} p_{2} g_{1}}\right) \\
& \left(\overline{p_{2} p_{1} p_{2} g_{2}}, \overline{p_{2} p_{2} p_{1} g_{1}}\right) \\
& \left(\overline{p_{2} p_{2} p_{1} g_{2}}, \overline{p_{2} p_{2} p_{2} g_{1}}\right)
\end{aligned}
$$


Table II. Binary expansions of the baby Mandelbrot sets of the single-spiral medallions when $j \leq 3$.

$$
\begin{array}{ccc}
\hline j=1 & j=2 & j=3 \\
\hline\left(\overline{b_{1} p_{1}}, \overline{b_{1} p_{2}}\right) & \left(\overline{b_{1} p_{1} p_{1}}, \overline{b_{1} p_{1} p_{2}}, \overline{b_{2} p_{2}}\right) & \left(\overline{b_{1} p_{1} p_{1} p_{1}}, \overline{b_{1} p_{1} p_{1} p_{2}}\right) \\
& \left(\overline{b_{2} p_{1} p_{1}}, \overline{b_{1} p_{2} p_{2}}\right) & \left(\overline{b_{1} p_{1} p_{2} p_{1}}, \overline{b_{1} p_{1} p_{2} p_{2}}\right) \\
& \left(\overline{b_{2} p_{2} p_{1}}, \overline{b_{1} p_{2} p_{1} p_{1}}, \overline{b_{1} p_{2} p_{1} p_{2}}\right) & \left(\overline{b_{1} p_{2} p_{2} p_{1}}, \overline{b_{1} p_{2} p_{2} p_{2}}\right) \\
& \left(\overline{b_{2} p_{1} p_{1} p_{1}}, \overline{b_{2} p_{1} p_{1} p_{2}}\right) \\
& \left(\overline{b_{2} p_{1} p_{2} p_{1}}, \overline{b_{2} p_{1} p_{2} p_{2}}\right) \\
& \left(\overline{b_{2} p_{2} p_{1} p_{1}}, \overline{b_{2} p_{2} p_{1} p_{2}}\right) \\
& \left(\overline{b_{2} p_{2} p_{2} p_{1}}, \overline{b_{2} p_{2} p_{2} p_{2}}\right)
\end{array}
$$


Table III. Binary expansions of the baby Mandelbrot sets of the non-spiral medallions when $j \leq 3$.

$$
\begin{array}{ccc}
\hline j=1 & j=2 & j=3 \\
\hline\left(\overline{b_{1} p_{1}}, \overline{b_{2} p_{2}}\right) & \left(\overline{b_{1} p_{1} p_{1}}, \overline{b_{2} p_{2} p_{2}}\right) & \left(\overline{b_{1} p_{1} p_{1} p_{1}}, \overline{b_{2} p_{2} p_{2} p_{2}}\right) \\
\left(\overline{b_{1} p_{2}}, \overline{b_{2} p_{1}}\right) & \left(\overline{b_{1} p_{1} p_{2}}, \overline{b_{2} p_{2} p_{1}}\right) & \left(\overline{b_{1} p_{1} p_{1} p_{2}}, \overline{b_{2} p_{2} p_{2} p_{1}}\right) \\
& \left(\overline{b_{1} p_{2} p_{1}}, \overline{b_{2} p_{1} p_{2}}\right) & \left(\overline{b_{1} p_{1} p_{2} p_{1}}, \overline{b_{2} p_{2} p_{1} p_{2}}\right) \\
& \left(\overline{b_{1} p_{2} p_{2}}, \overline{b_{2} p_{1} p_{1}}\right) & \left(\overline{b_{1} p_{1} p_{2} p_{2}}, \overline{b_{2} p_{2} p_{1} p_{1}}\right) \\
& \left(\overline{b_{1} p_{2} p_{1} p_{1}}, \overline{b_{2} p_{1} p_{2} p_{2}}\right) \\
& \left(\overline{b_{1} p_{2} p_{1} p_{2}}, \overline{b_{2} p_{1} p_{2} p_{1}}\right) \\
& \left(\overline{b_{1} p_{2} p_{2} p_{1}}, \overline{b_{2} p_{1} p_{1} p_{2}}\right) \\
& \left(\overline{b_{1} p_{2} p_{2} p_{2}}, \overline{b_{2} p_{1} p_{1} p_{1}}\right)
\end{array}
$$




\section{Figure captions}

Fig. 1. External rays and external arguments in the Mandelbrot set.

Fig. 2. (a) General view of the Mandelbrot set. (b) Magnification of the midget $(\overline{.0011}, \overline{0100})$ showing its shrubs (gross lines) and tendrils (thin lines).

Fig. 3. A detail of the tendrils in the cusp of the midget $(\overline{.0011}, \overline{.0100})$. The midgets of the tendrils are surrounded with decorations.

Fig. 4. Tendrils of the midget $(\overline{.0011}, \overline{.0100})$. (a) Near the tangent point of the cardioid with the period-2 disc. (b) Near the tangent point of the cardioid with a period-3 disc.

Fig. 5. Multiple-spiral medallions in the Mandelbrot set near the midget $(\overline{.0011}, \overline{.0100})$.

Fig. 6. Multiple-spiral medallions with low period central baby Mandelbrot sets. The parent and the gene are, respectively, $\left(. \overline{p_{1}}, \overline{p_{2}}\right)=(\overline{.0011}, \overline{0100})$ and $\left(\overline{g_{1}}, \overline{g_{2}}\right)=(\overline{.001}, . \overline{010})$. (a) Non-spiral medallions. (b) Single-spiral medallions. (c) Double-spiral medallions. (d) Triple-spiral medallions.

Fig. 7. Examples of the parent and the gene of a multiple-spiral medallion. (a) The parent is in a primary shrub. (b) The parent is in a secondary shrub.

Fig. 8. A sketch of the ordering of the binary expansions pairs of the central baby Mandelbrot sets of multiple-spiral medallions.

Fig. 9. External rays and binary expansions of the central baby Mandelbrot sets of multiple-spiral medallions, $i \leq 3$, surrounding the parent $\left(. \overline{p_{1}}, \overline{p_{2}}\right)=(\overline{.0011}, \overline{0100})$. The gene is $\left(\overline{g_{1}}, \overline{g_{2}}\right)=(\overline{.001}, \overline{.010})$.

Fig. 10. Location of the baby Mandelbrot sets with binary expansions $\overline{. b_{*} \underbrace{p_{*} p_{*} \ldots p_{*}}_{j}}$ $(j \leq 3)$ inside of multiple-spiral medallions. (a) Non-spiral medallion; (b) Single-spiral medallion; (c) Double-spiral medallion; (d) Triple-spiral medallion. 


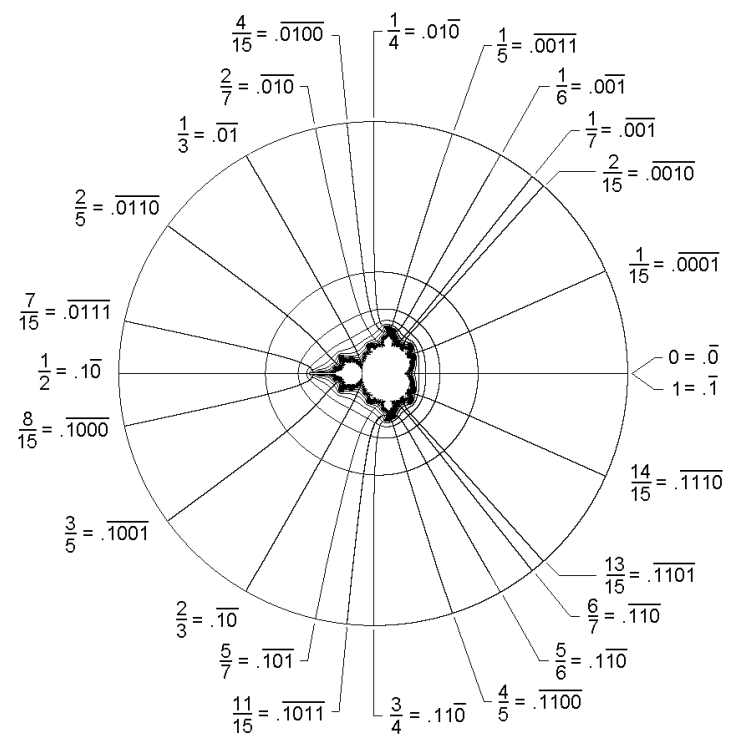

Fig. 1. 


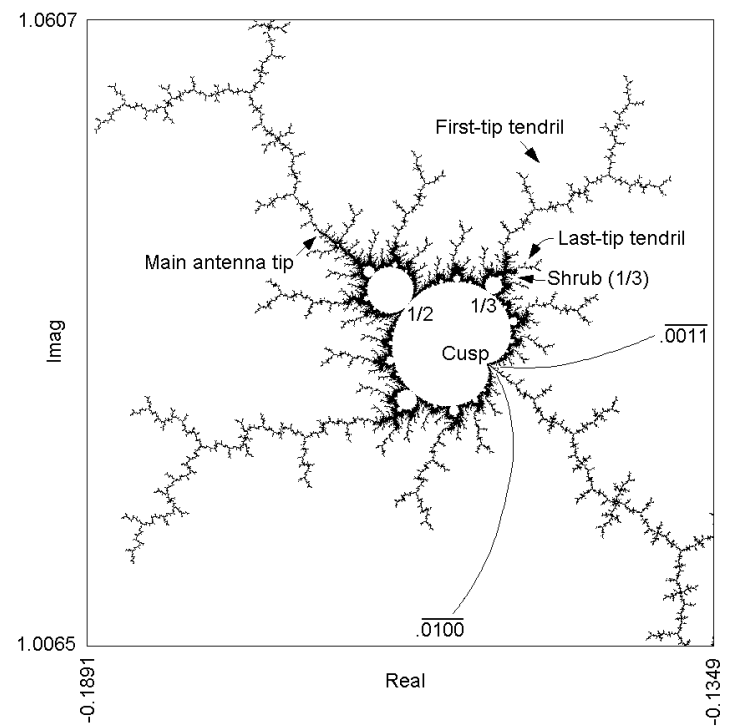

(b)

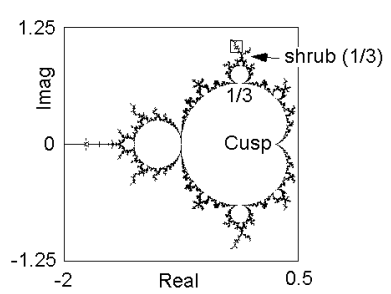

(a)

Fig. 2. 


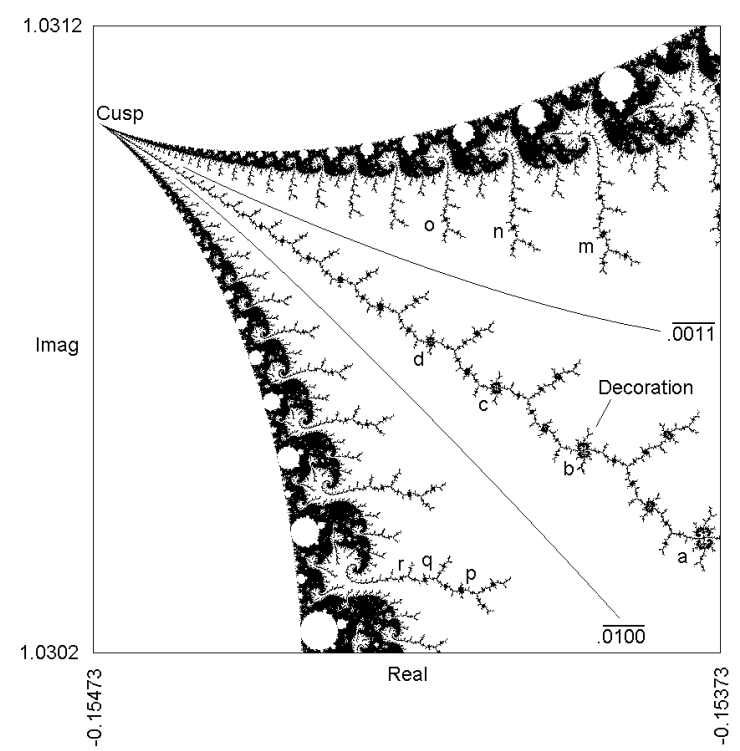

Fig. 3. 


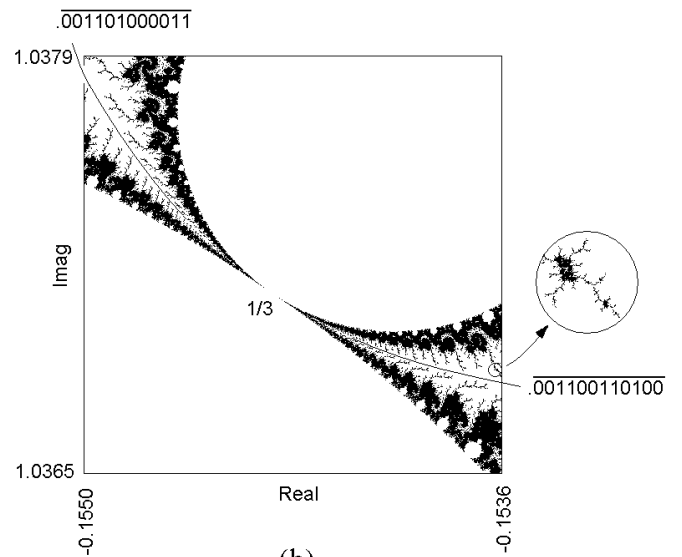

(b)

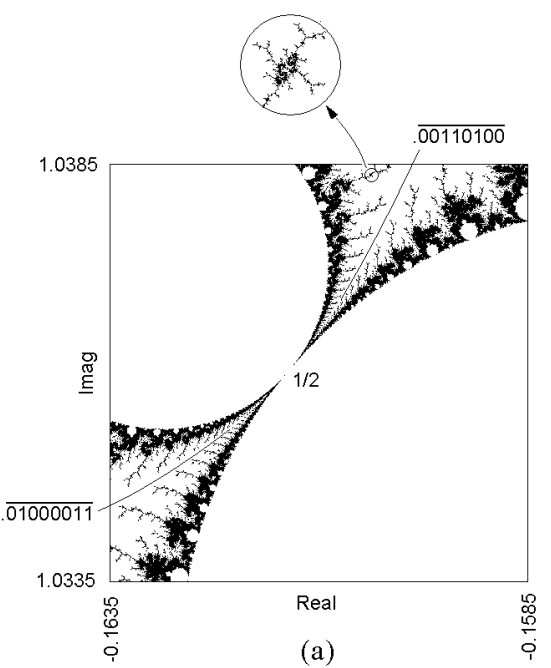

Fig. 4. 


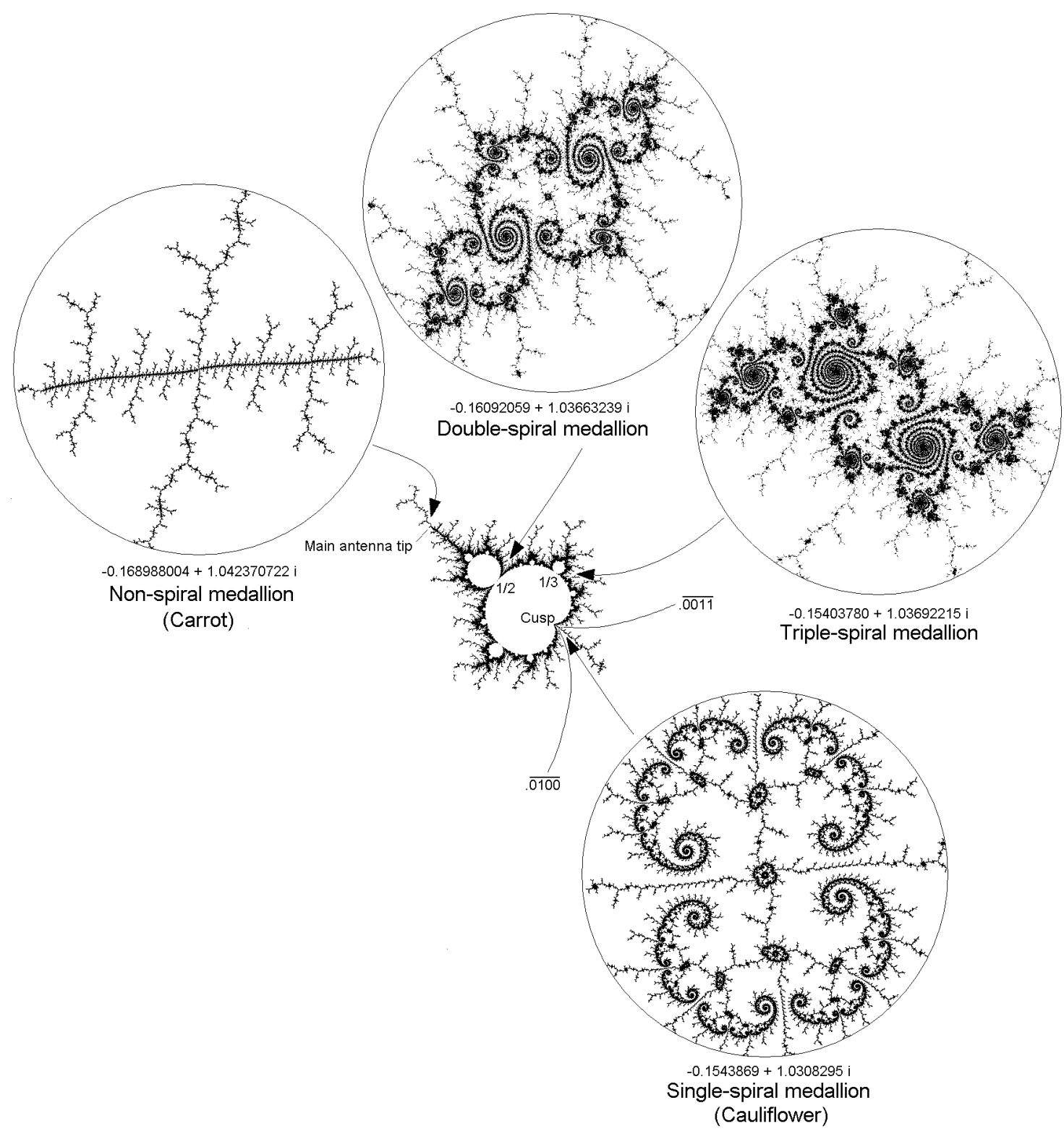

Fig. 5 
(a)

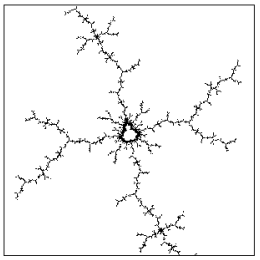

$\overline{p_{1} g_{2}}, \overline{p_{2} g_{1}}$

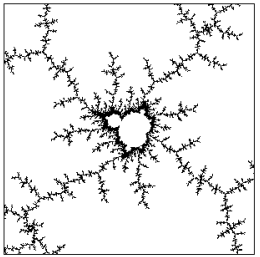

$\overline{p_{1} g_{1}}, \overline{p_{2} g_{2}}$

(c)

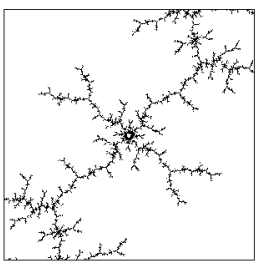

$\overline{p_{1} p_{2} p_{1} g_{2}}, \overline{p_{1} p_{2} p_{2} g_{1}}$

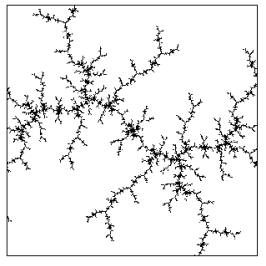

$\overline{p_{1} p_{1} p_{2} p_{1} g_{2}}, \overline{p_{1} p_{1} p_{2} p_{2} g_{1}}$

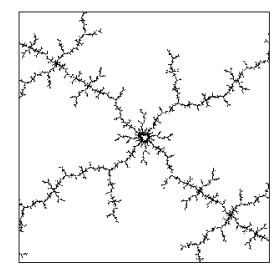

$\overline{p_{1} p_{2} g_{2}}, \overline{p_{2} p_{1} g_{1}}$
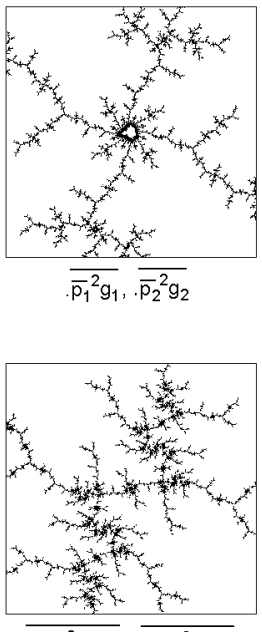

$\overline{. \bar{p}_{1} p_{2}^{2} p_{1} g_{2}}, \overline{. \bar{p}_{1} p_{2}^{2} p_{2} g_{1}}$

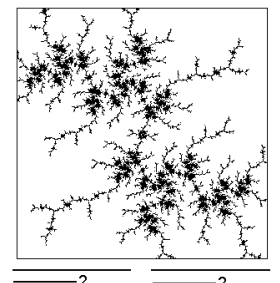

${\overline{p_{1} p_{1} p_{2}}}^{2} p_{1} g_{2}, .{\overline{p_{1} p_{1} p_{2}}}^{2} p_{2} g_{1}$

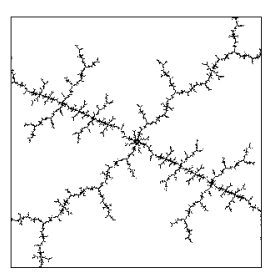

$\overline{p_{1} \bar{p}_{2}^{2} g_{2}}, \overline{p_{2} \bar{p}_{1}^{2} g_{1}}$

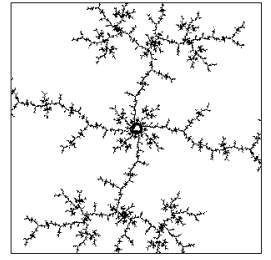

$\overline{\bar{p}_{1}^{3} g_{1}}, \overline{\bar{p}_{2}^{3} g_{2}}$

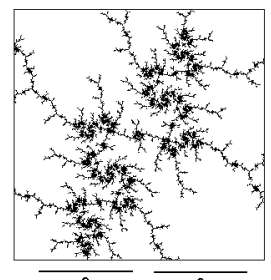

$\overline{.{\overline{p_{1} p_{2}}}^{3} p_{1} g_{2}}, \overline{{\overline{p_{1} p_{2}}}^{3} p_{2} g_{1}}$

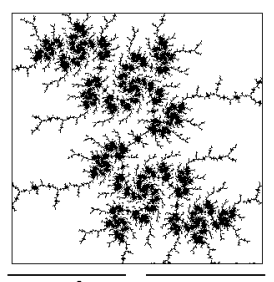

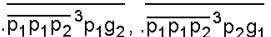
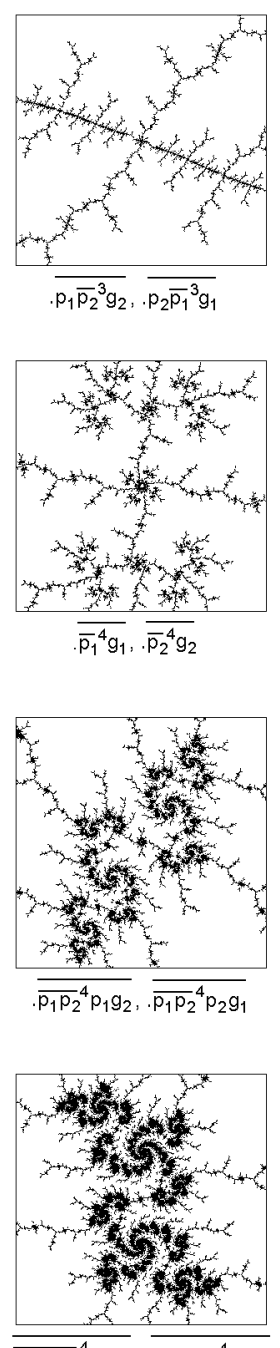

$\overline{\overline{p_{1} p_{1} p_{2}}{ }^{4} p_{1} g_{2}}, \overline{\overline{p_{1} p_{1} p_{2}}{ }^{4} p_{2} g_{1}}$

Fig. 6 

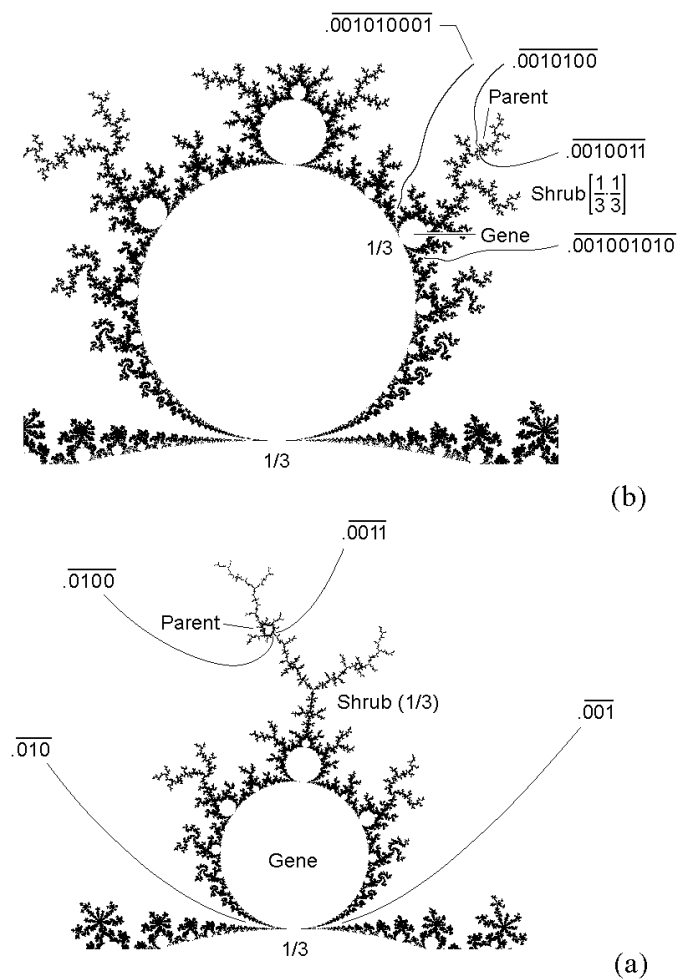

Fig. 7. 


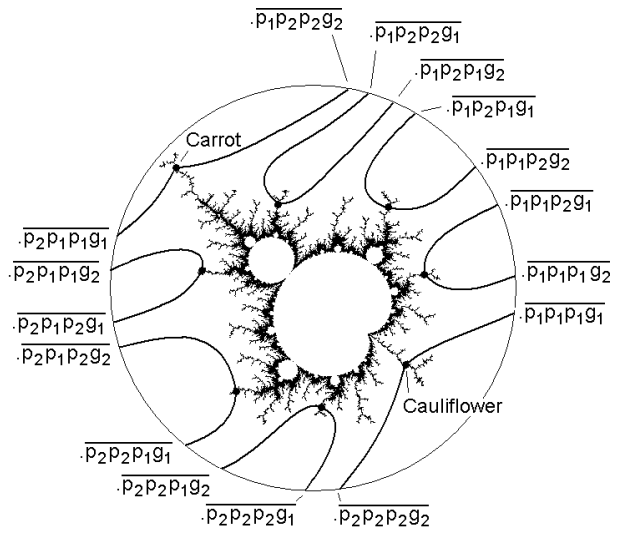

Fig. 8. 


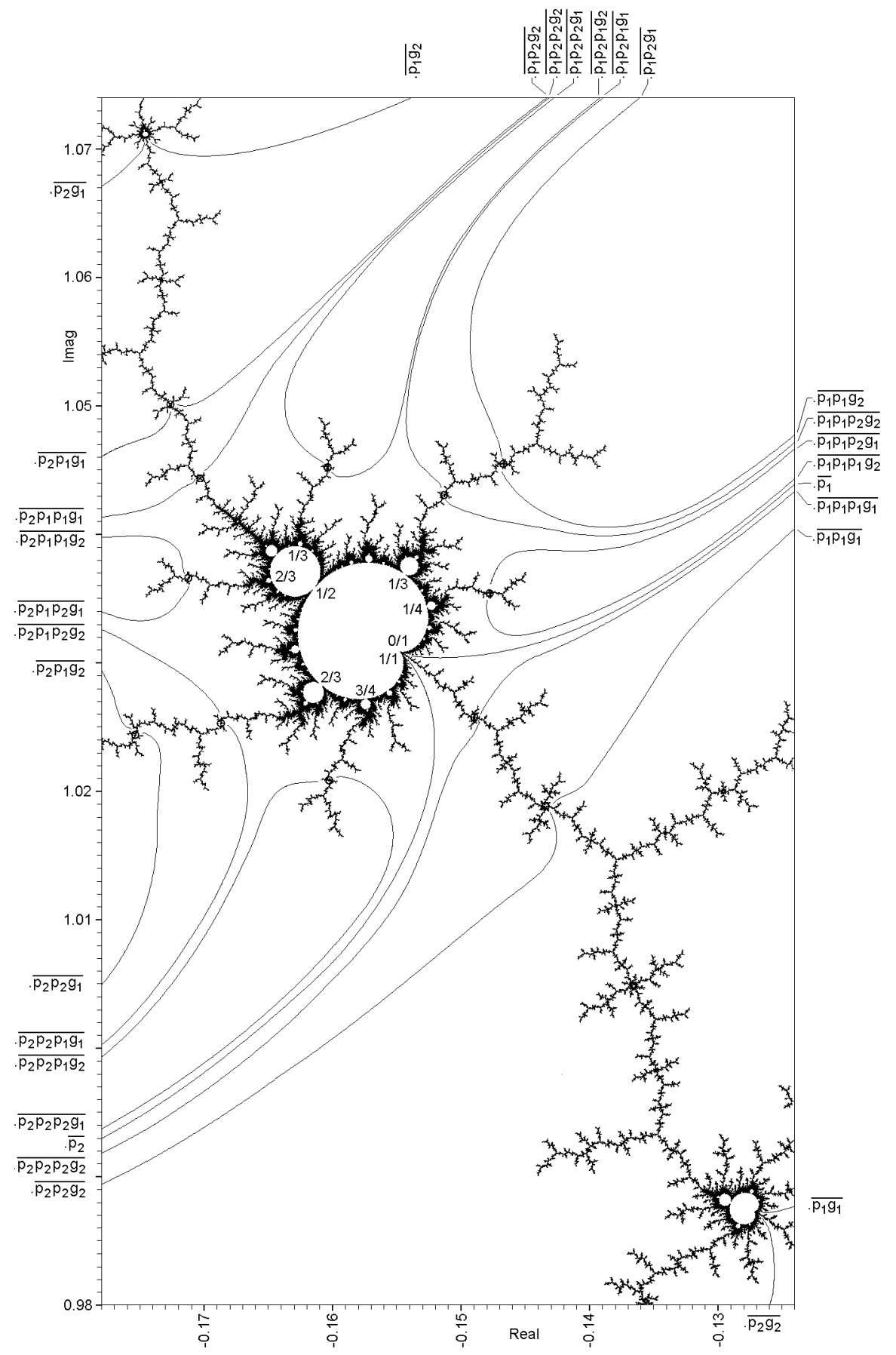

Fig. 9. 


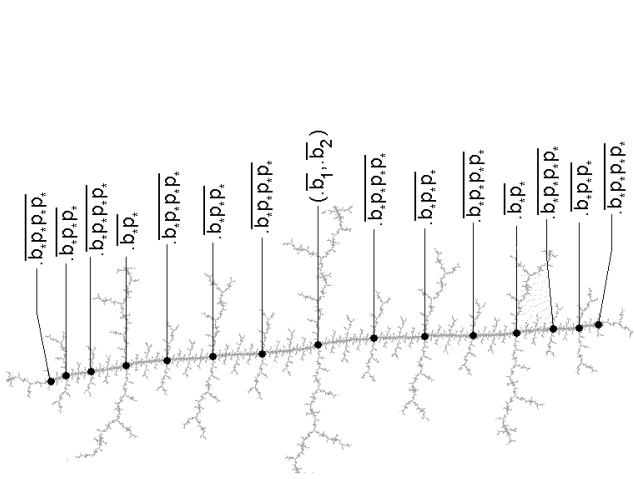

(a)

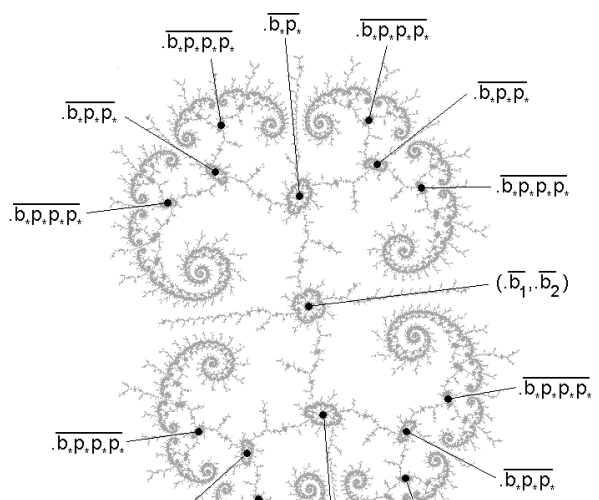

(b)

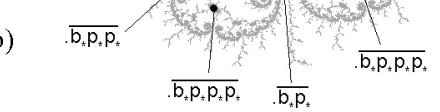

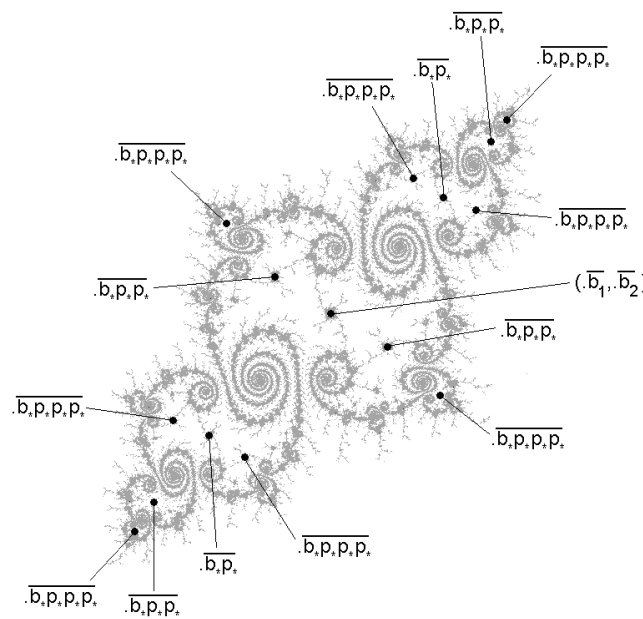

(c)

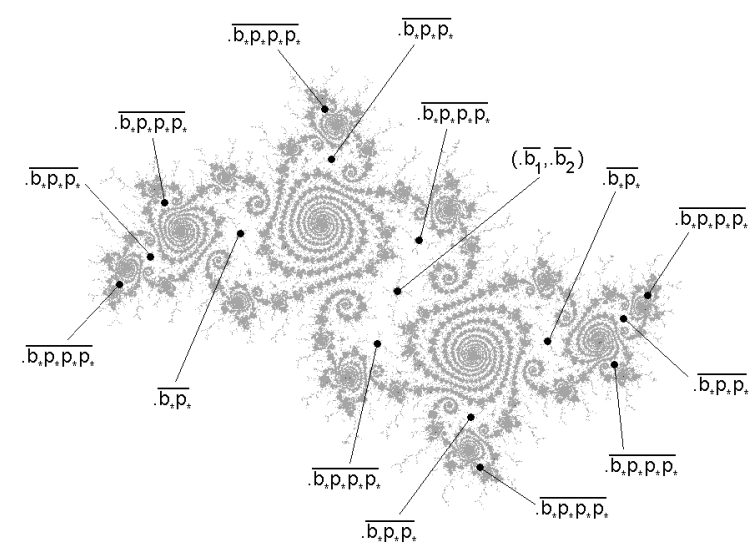

(d)

Fig. 10. 